\title{
Endoparasite prevalence and infection risk factors among cats in an animal shelter in Estonia
}

\author{
Ants Tull ${ }^{1}$, Epp Moks ${ }^{1,2}$ and Urmas Saarma ${ }^{1}$ \\ ${ }^{1}$ Department of Zoology, Institute of Ecology and Earth Sciences, University of Tartu, Estonia; \\ ${ }^{2}$ Veterinary and Food Laboratory, Department of bacteriology-pathology, Tartu, Estonia
}

\begin{abstract}
Cats are important hosts for different zoonotic parasites that can be hazardous to human health. To date, few studies have attempted to identify the factors affecting parasitic infections in shelter animals. This study aims to analyse the presence of endoparasites in shelter cats in Tartu, Estonia, and identify factors affecting endoparasite prevalence and intensity. The risk factors considered were age, location (urban $v s$ rural cats) and time spent in shelter. In total, 290 faecal samples were collected from cats at an animal shelter in 2015-2016 and investigated for endoparasites using the concentration flotation technique. In total, 138 shelter cats (47.6\%) were infected with endoparasites and their overall prevalence was: Toxocara cati (36.6\%), Cystoisospora spp. (12.4\%), Taeniidae gen. sp. (4.1\%), Toxoplasma gondii/Hammondia hammondi (3.4\%), Eucoleus aerophilus (2.1\%), Cryptosporidium spp. (2.1\%), Ancylostoma sp. (0.7\%) and Giardia sp. (0.7\%). Coinfections occurred in 38 cats (13.1\%) most frequently of T. cati and Cystoisospora spp. (4.5\%), Cystoisospora spp. and T. gondii/H. hammondi (2.1\%). Where species identification of cestode and nematode samples was not possible according to morphology, genetic analysis of the mitochondrial coxl gene was carried out. DNA was successfully analysed for 6 out of 13 samples that required genetic identification, revealing Ancylostoma tubaeforme in one nematode sample and Hydatigera taeniaeformis in five cestode samples. Cats from rural areas had significantly higher endoparasite prevalence than cats from urban areas. Helminth prevalence decreased to some extent due to anthelmintic treatment in cats available for adoption (held $\geq 15$ days in the shelter), whereas the prevalence of infection with protists increased significantly in these animals. It is important to note that the analysis revealed lower infection intensity for quarantine cats (held 1-14 days in the shelter) compared with cats available for adoption. The relatively high prevalence of endoparasites (including zoonotic) in shelter cats ready for adoption suggests that current anthelminthic procedures require improvements.
\end{abstract}

Keywords: rural cats, shelter cats, Toxocara cati, Hydatigera taeniaeformis, urban cats, shelter management

This article contains supporting information online at http://folia.paru.cas.cz/suppl/2021-68-010.pdf

Free-ranging cats host a wide range of zoonotic endoparasites (Overgaauw et al. 2009, Otranto et al. 2015, Kostopoulou et al. 2017) from protists to helminths, e.g., Cryptosporidium felis Iseki, 1979, Toxoplasma gondii (Nicolle et Manceaux, 1908), Giardia duodenalis (Lambl, 1859) assemblage A, Toxocara cati (Schrank, 1788), Echinococcus multilocularis Leuckart, 1863, Hydatigera taeniaeformis Batsch, 1786 and Eucoleus aerophilus (Creplin, 1839) (Leoni et al. 2003, Deplazes et al. 2004, Gates and Nolan 2009, Suzuki et al. 2011, Wyrosdick et al. 2017). These zoonotic endoparasites pose a serious threat to human health, especially to children who often come into close contact with cats and play in areas that may be contaminated by parasite eggs and oocysts (Talvik et al. 2006, Schurer et al. 2013, Lassen et al. 2016). In general, studies to date suggest that the main risk factors associated with endoparasite infection in cats are a free-ranging lifestyle, living in a rural environ- ment and a young age (Mircean et al. 2010, Becker et al. 2012, Nijsse et al. 2016, Zottler et al. 2019). Furthermore, hunting, consumption of raw or undercooked meat and older age are the key risk factors for $T$. gondii infection, and being from multi-cat households or cattery is a risk factor for $G i$ ardia sp. (Jokelainen et al. 2012, Deksne et al. 2013, Must et al. 2015, Blasco et al. 2017).

Previous studies have demonstrated a high prevalence of zoonotic endoparasites in stray cats (Becker et al. 2012, Villeneuve et al. 2015, Blasco et al. 2017). Information on the prevalence of feline gastrointestinal parasites, especially those of zoonotic importance, is important for protecting shelter staff and cat owners, and represents fundamental information for veterinarians and the authorities responsible for the controlling and preventing zoonotic diseases. However, no studies on the fauna of endoparasites in shelter cats have been carried out in Estonia. This study aims to: i) de- 
termine endoparasite prevalence and identity in cats at the Tartu Animal Shelter, which is one of the largest shelters in southern Estonia; and ii) identify the factors affecting endoparasite prevalence and intensity of infection in shelter cats.

\section{MATERIALS AND METHODS}

\section{The animal shelter}

During the study period, over a thousand captured cats from nearby counties and towns were hosted at the Tartu Animal Shelter (58.390684N, 26.746292E) (Statistics of Tartu Animal Shelter 2019). Most of the captured cats were stray or abandoned, and only a minority of cats were brought in by private individuals (these were excluded from the analysis). On the first or second day after arrival at the shelter, cats are treated with Vitaminthe ${ }^{\circledR}$ (Virbac Laboratories SA, France), which is an oral, broad-spectrum anthelmintic in paste form for cats and dogs. This drug contains two active ingredients, of which the niclosamide $(120 \mathrm{mg} /$ $\mathrm{kg})$ acts against tapeworms and oxibendazol $(15 \mathrm{mg} / \mathrm{kg})$ against the adult and larval stages of roundworms, whipworms and hookworms. If clinical signs related to protozoan infection occur (diarrhea, vomiting, weight loss, lethargy etc.), treatment with metronidazole $(50 \mathrm{mg} / \mathrm{kg})$ is used against protozoa. According to the shelter policy, cats are kept in quarantine for 14 days, after which period they are available for adoption. Most of the adult cats are held in separate cages, though some are held together in groups consisting of 2-4 individuals. Kittens are located with females or in separate smaller cages. During the daily cleaning process, some kittens and adults are let out of their cages to socialise and play with one another. All ethical requirements stipulated in Estonian law were met by the shelter.

\section{Sample collection and faecal examination}

Shelter cat faecal samples $(\mathrm{N}=290)$ were collected from August 2015 to October 2016. Faecal samples were collected in the morning up to three times a week from randomly selected cats before cleaning the litter trays. All samples were placed into a separate plastic bag and tagged with the unique ID given to each cat on arrival at the shelter, which also helped to prevent resampling the same animals. Pooled samples were also taken from kitten (belonging to the same litter) cages. Collected faecal samples were deep frozen at $-80^{\circ} \mathrm{C}$ for a minimum of seven days to inactivate eggs of Echinococcus multilocularis, which is endemic in Estonia (Moks et al. 2005, Laurimaa et al. 2015a, b). After thawing the samples, the sodium chloride $(\mathrm{NaCl}$, specific gravity $=1.2 \mathrm{~g} / \mathrm{cm}^{3}$ ) concentration flotation technique (Roepstorff and Nansen 1998) was used, followed by parasite egg counting in each McMaster chamber and identification based on morphological characteristics (Pavlásek and Ryan 2007, Khatat et al. 2016, Dubey 2018, Tokiwa et al. 2018, Greenwood 2020). The total number of eggs per gram of faeces (EPG) was calculated by multiplying the number of eggs in both sides of the chamber by a coefficient of 20 (Roepstorff and Nansen 1998). Positive samples with endoparasite eggs in the McMaster chamber were washed with distilled water droplets (using a $3 \mathrm{ml}$ plastic pipette) into 2 $\mathrm{ml}$ tubes filled with $70 \%$ ethanol solution and stored at $-20{ }^{\circ} \mathrm{C}$. Endoparasite prevalence was defined as the proportion of all parasitic oocysts/eggs in faecal samples, and infection intensity was determined as the number of oocysts/eggs per gram in a sample.

\section{Molecular diagnostics}

Molecular methods were used to identify eggs of tapeworms (species of Taenia Linnaeus, 1758) and nematodes (Ancylostoma Dubini, 1843) to the species level. Single eggs of these cestode and nematode taxa were first pipetted onto micro slides into distilled water droplets and subsequently isolated with a pipette into $1.5 \mathrm{ml}$ tubes for DNA analysis. DNA extraction and PCR were performed in a laboratory dedicated to analysis of samples with low quantities of DNA. The DNA was extracted using the High Pure PCR Template Preparation Kit (Roche Diagnostics $\mathrm{GmbH}$, Germany) according to the manufacturer's instructions. Specific new primers were designed for the mtDNA COI gene for both cestodes (CesCox1F-TGATCCGTTAGGTGGTGGTGA and CesCox2R-ACCCTAACGACATAACATAATGAAAATG, yielding a PCR product of $506 \mathrm{bp}$ in length) and nematodes (NemCox1F-CTATGATTGGTGGTTTTGGTAATTGA and NemCox1R-ATAATGAAAATGACTAACAACATAATAAG; 917 bp). The PCR and sequencing procedures followed Saarma et al. (2009). Briefly, PCR was performed in a total volume of $20 \mu 1$ containing $3 \mu \mathrm{l}$ of purified genomic DNA, 5 pmol of each primer, 1 X BD Advantage 2 PCR buffer (BD Biosciences, Franklin Lakes, NJ, USA), 1U BD Advantage 2 Polymerase mix and 0.2 mM dNTP (Thermo Fisher Scientific, Waltham, MA, USA). PCR amplifications without DNA were used as negative controls. The PCR conditions were as follows: 1 min-denaturing step at $95^{\circ} \mathrm{C}$, followed by 10 cycles of $20 \mathrm{~s}$ at $95^{\circ} \mathrm{C}, 45 \mathrm{~s}$ at $60^{\circ} \mathrm{C}$ (the annealing temperature reduced by $0.5^{\circ} \mathrm{C}$ in each step) and 2 min at 68 ${ }^{\circ} \mathrm{C}$, followed by 27 cycles of $20 \mathrm{~s}$ at $95{ }^{\circ} \mathrm{C}, 45 \mathrm{~s}$ at $55^{\circ} \mathrm{C}$ and 2 $\min$ at $68{ }^{\circ} \mathrm{C}$. Of the $20 \mu \mathrm{l}$ PCR products, $10 \mu \mathrm{l}$ were examined on $1.4 \%$ agarose gel electrophoresis and the remaining $10 \mu \mathrm{l}$ were purified with the mix of $1 \mathrm{U}$ of FastAP Phosphatase and $1 \mathrm{U}$ of Exonuclease I (Thermo Fisher Scientific). For all positive samples both DNA strands were sequenced using the same primers as for PCR. Quality checking and sequence assembly were conducted manually using the program CodonCode v6.0.2, and Nucleotide BLAST was used to search for homologous sequences.

\section{Statistical analysis}

Information about the time and initial capture location, sex, anthelmintic treatment and age was written on an ID-card for each cat brought to the animal shelter. The dependent and independent variables were divided into seven categories that are explained in Table 1. Proportions were compared using Chisquared tests of independence to determine predictor variables associated with overall endoparasite prevalence (Table 2). If one or more cells in the $2 \times 2$ tables had expected values of less than 5, Fisher's exact test was used. Statistical modeling was used to evaluate risk factors (location, age, time and sex; Table 2) associated with overall endoparasite prevalence $(\mathrm{N}=290)$, coinfection prevalence $(\mathrm{N}=190)$ and infection intensity $(\mathrm{N}=290)$. We also estimated how these aforementioned risk factors associate with the prevalence and intensity of individual parasite taxa (Table 2). Furthermore, a distinct group of potentially directly transmittable endoparasites was formed consisting of Toxoplasma gondii/Hammondia hammondi (Frenkel et Dubey, 1975), Cryptosporidium spp., Giardia sp., Cystoisospora spp., Toxocara cati and Eucoleus aerophilus (Online Resource 4).

Because of the large standard errors, Firth 's bias reduced logistic regression (R package 'logistf' - Heinze and Ploner 2018) was 


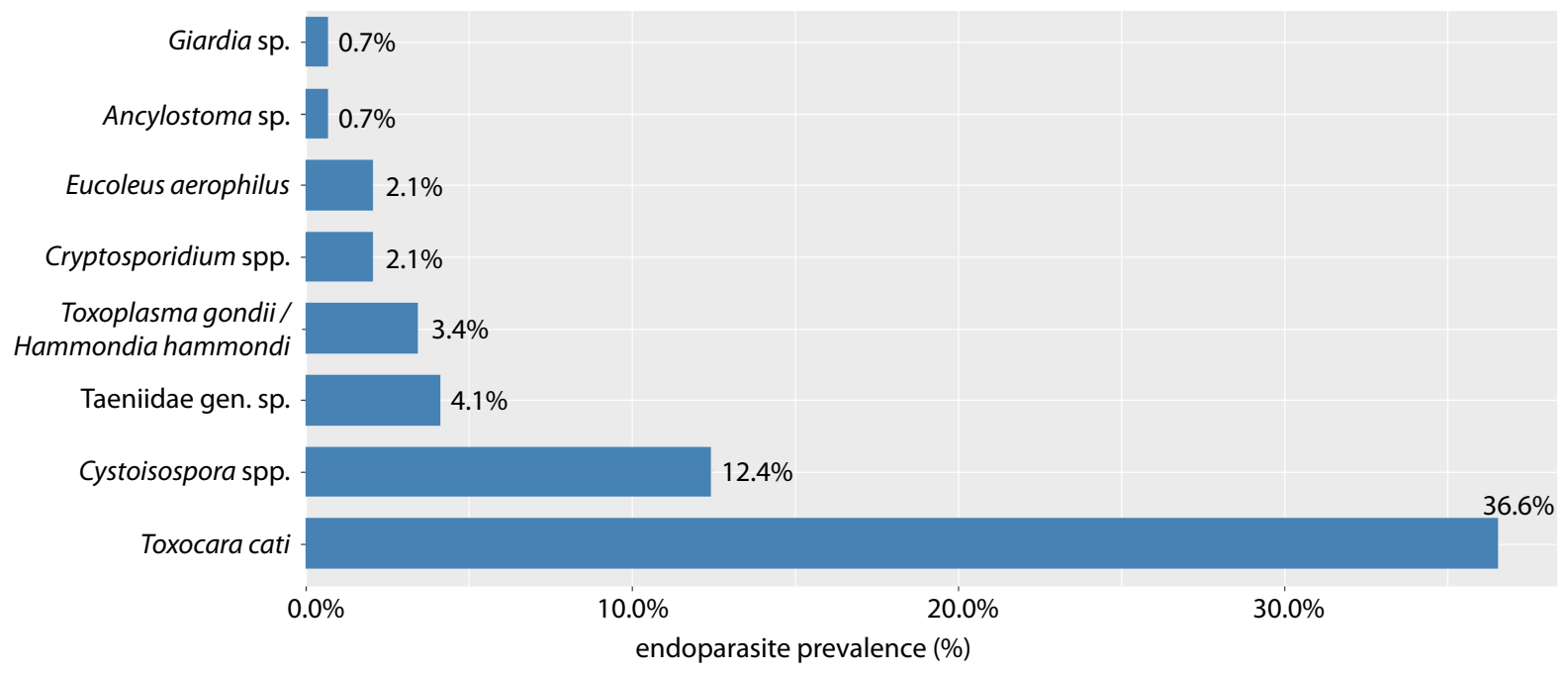

Fig. 1. Total endoparasite prevalence among shelter cats from an animal shelter in Tartu, Estonia

used to model the relationship between coinfection prevalence and a set of explanatory variables including age, location and the time spent in the shelter. The variable 'sex' was dropped from the overall models of endoparasite prevalence and intensity due to nonsignificant differences between the sexes. Generalised linear models (package "glmmTMB", Brooks et al. 2017) with a binomial error distribution were used for evaluating overall and single endoparasite prevalence. Models with a negative binomial error distribution were used for assessing the factors influencing endoparasite intensity among shelter cats. Statistical analyses were performed in R (R Core Team 2020). Models were compared using the Akaike information criterion corrected for small samples (AICc) (Burnham and Anderson 2004). Package "MuMIn" (Barton 2019) was used for conducting model selection and model averaging. Here, we describe only models with the highest Akaike weight wi(AIC) $(\triangle \mathrm{AICc}<2)$. Models with lower weights and statistics for intensity and (co)infection models can be found in Online Resource 1-4.

\section{RESULTS}

\section{Parasite identification, prevalence, coinfection and intensity}

Parasitological examination revealed that $47.6 \%$ (138/290; 95\% CL 41.8-53.4) of the shelter cats were infected with endoparasites (Table 3). Single infections were significantly $(\mathrm{p}<0.0001)$ more common $(34.5 \%)$ than infections with two $(11.7 \%)$, three $(1.0 \%)$ or four $(0.3 \%)$ endoparasite species. Overall, coinfection occurred in $13.1 \%$ (38/290; 95\% CL 9.2-17.0) of examined cats. More than half $(91 / 160 ; 95 \%$ CL $49.1-64.6)$ of cats infected with endoparasites were from rural areas, compared to $36.2 \%$ (47/130; 95\% CL 27.8-44.5) of the urban cats (Table 3). There was a significant difference in infection prevalence between the rural and urban cats $(p=0.0006)$.

On the basis of morphological and genetic analyses, we identified Ancylostoma tubaeforme (Zeder, 1800), Toxocara cati, Eucoleus aerophilus, Giardia sp., Cryptosporidium spp., Toxoplasma gondii/Hammondia hammondi, Cystoisospora spp., Hydatigera taeniaeformis and Taenii- dae gen. sp. Over a third (36.6\%; 95\% CL 31.0-42.1) of the examined shelter cats excreted eggs of zoonotic $T$. cati (106/290) (Table 2). Mean infection intensity ranged from 20 to 5,195 EPG/OPG (Table 2).

Highest prevalence was estimated for $T$. cati (36.6\%), followed by Cystoisospora spp. (12.4\%), Taeniidae gen. sp. (4.1\%), T. gondii/H. hammondi (3.4\%), E. aerophilus (2.1\%), Cryptosporidium spp. (2.1\%), Ancylostoma sp. $(0.7 \%)$ and Giardia sp. $(0.7 \%)$ (Fig. 1). The highest coinfection prevalence was revealed for T. cati/Cystoisospora spp. (4.5\%), Cystoisospora spp./T. gondii/H. hammondi (2.1\%), T. cati/E. aerophilus $(1.4 \%)$ and T. cati/ Taeniidae gen. sp. (1.0\%) (Fig. 2).

\section{Risk factors associated to endoparasite infection}

The best endoparasite prevalence model (model average of models with $\triangle \mathrm{AICc}<2$ ) revealed that urban cats had lower parasite infection prevalence than rural cats $\left(\beta_{\mathrm{UR}}\right.$ $\left.{ }_{\text {BAN }}=-0.9, \mathrm{SE}=0.3, \mathrm{p}<0.001\right)$. Quarantine cats $(1-14$ days in shelter) had lower endoparasite infection prevalence $\left(\beta_{\text {QUARANTINE }}=-0.5, \mathrm{SE}=0.3, \mathrm{p}=0.03\right)$ compared with cats ready for adoption ( $\geq 15$ days in the shelter) (Online Resource 3).

Rural cats in quarantine had significantly higher endoparasite infection prevalence with directly transmittable endoparasites than urban cats in quarantine $\left(\chi^{2}=5.5\right.$; $\mathrm{p}=0.01$; all Chi-squared tests between potentially directly transmitted endoparasites and time spent in shelter by location are available in Online Resource 4).

Young cats had significantly higher endoparasite infection prevalence with $T$. cati $\left(\beta_{\mathrm{YOUNG}}=0.7, \mathrm{SE}=0.3\right.$, $\mathrm{p}=0.01)$ and with helminths $\left(\beta_{\mathrm{YOUNG}}=0.5, \mathrm{SE}=0.3\right.$, $\mathrm{p}=0.04$ ) than adult cats (Table 2). Young cats had significantly lower infection prevalence with $T$. gondii/H. hammondi than adult cats $\left(\beta_{\mathrm{YOUNG}}=-2.8, \mathrm{SE}=1.5, \mathrm{p}=0.003\right)$ (Table 2). Urban cats had significantly lower endoparasite infection prevalence with Taeniidae gen. $\mathrm{sp} .\left(\beta_{\mathrm{URBAN}}=-2.1\right.$, $\mathrm{SE}=1.1, \mathrm{p}=0.04)$, T. $\operatorname{cati}\left(\beta_{\mathrm{URBAN}}=-1.0, \mathrm{SE}=0.3, \mathrm{p}<\right.$ $0.001)$ and with total helminths $\left(\beta_{\text {URBAN }}=-1.0, \mathrm{SE}=0.3, \mathrm{p}\right.$ $<0.001$ ) than rural cats (Table 2). Endoparasite infection 
Table 1. Predictor variables used in the logistic regression analyses.

\begin{tabular}{|c|c|c|c|}
\hline Predictor variable & Response variable & Predictor categories & Explanation \\
\hline Location & - & rural, urban & $\begin{array}{l}\text { stray cats captured by shelter staff in urban or rural environments and taken to the } \\
\text { shelter }\end{array}$ \\
\hline - & Infection occurrence & 0 or 1 & no infection (0) or infected (1) with endoparasites \\
\hline Sex & - & male, female & male or female cat \\
\hline Age & - & young, adult & less than a year or over one year old cats \\
\hline Time & - & $1-14$ days, $\geq 15$ days & $\begin{array}{l}\text { Time spent in the shelter: quarantine group (1-14 days) and cats ready for adoption } \\
(\geq 15 \text { in the shelter) }\end{array}$ \\
\hline - & Coinfection occurrence & 0 or 1 & Cats infected with none ( 0$)$ and more than one (1) endoparasite species \\
\hline- & Infection intensity & $0-46,020$ & The sum of parasite eggs/oocysts counted in both McMaster chambers (EPG/OPG) \\
\hline
\end{tabular}

prevalence with Cystoisospora spp. $\left(\beta_{\text {QUARANTINE }}=-1.3\right.$, $\mathrm{SE}=0.4, \mathrm{p}=0.001)$ and with total protozoa $\left(\beta_{\text {QUARAN }}\right.$ $\left.{ }_{\mathrm{TINE}}=-0.8, \mathrm{SE}=0.3, \mathrm{p}=0.01\right)$ was significantly less common in quarantine cats than in cats ready for adoption (Table 2). Infection prevalence with Cryptosporidium spp. $\left(\beta_{\text {QuARAntine }}=2.3, \mathrm{SE}=1.5, \mathrm{p}=0.03\right)$ and with $E$. aerophilus $\left(\beta_{\text {QUARANTINE }}=2.3, \mathrm{SE}=1.4, \mathrm{p}=0.03\right.$ ) was more common in quarantine cats than in cats ready for adoption (Table 2). Male cats had significantly lower infection with E. aerophilus $\left(\beta_{\mathrm{MALE}}=-2.3, \mathrm{SE}=1.4, \mathrm{p}=0.04\right)$ compared with female cats (Table 2).

Coinfection prevalence with endoparasites was significantly lower among urban than rural cats $\left(\beta_{\text {URBAN }}=-1.3\right.$, $\mathrm{SE}=0.4, \mathrm{p}=0.0005)$ (Online Resource 2). Models also indicated that young cats were more frequently coinfected with multiple endoparasite species, compared with adult cats $\left(\beta_{\text {YOUNG }}=0.3, \mathrm{SE}=0.4, \mathrm{p}=0.4\right)$. Similarly, a non-significant trend suggested that quarantine cats less frequently exhibited coinfections, compared with cats ready for adoption $\left(\beta_{\text {QUARANTINE }}=-0.4, \mathrm{SE}=0.4, \mathrm{p}=0.3\right)$ (Online Resource 2).

The best infection intensity model revealed 2.7 times higher infection intensity for young shelter cats $\left(\beta_{\text {YOUNG }}=1.0, \mathrm{SE}=0.4, \mathrm{p}=0.02\right)$ than for adults (Online Resource 1). The intensity model showed lower infection intensity for quarantine cats $\left(\beta_{\text {QUARANTINE }}=-0.6, \mathrm{SE}=0.4\right.$, $\mathrm{p}=0.09)$ compared with cats available for adoption (Online Resource 1).

\section{Molecular diagnostics}

DNA was successfully extracted and amplified from 6 of 13 samples. Of these, five samples were identified as $H$. taeniaeformis (initially identified as Taeniidae gen. sp.) and one as Ancylostoma tubaeforme (initially a hookworm). The $H$. taeniaeformis sequences showed $>99 \%$ identity match according to the Nucleotide BLAST search and were divided into three haplotypes (Online Resource 5). The sequence of $A$. tubaeforme had $98.4 \%$ identity match. All mtDNA coi sequences are available in GenBank under accession codes MT407624-MT407626 for different haplotypes of $H$. taeniaeformis and MT407597 for $A$. tubaeforme.

\section{DISCUSSION}

The overall prevalence of endoparasite infection among shelter cats estimated in this study $(47.6 \%)$ was higher than the corresponding figures reported by the majority of analogous studies. For example, in Australia overall prev- alence was 8.3\% (McGlade et al. 2003), in Switzerland 21.8\% (Zottler et al. 2019), in Canada 31.8\% (Villeneuve et al. 2015) and in Germany 33.6\% (Becker et al. 2012). However, a higher prevalence rate was recorded in a shelter in Catalonia, Spain (57\%, Blasco et al. 2017). It is difficult to pinpoint the exact reasons for this variation, but likely candidates are the overall levels of hygiene and the efficacy of anthelmintic treatments and/or consumption of anthelmintics. In Estonia, the high parasite prevalence may be characteristic of the Tartu Animal Shelter, and further studies are needed to obtain broader estimates for animal shelters throughout Estonia.

\section{Helminths with zoonotic potential}

Over a third of examined shelter cats in our study $(36.6 \%)$ excreted parasitic stages of zoonotic Toxocara cati - one of the most prevalent zoonotic gastrointestinal parasite worldwide (Gracenea et al. 2009, Becker et al. 2012, Loftin et al. 2019), causing toxocariasis in humans, mainly in young children (Despommier 2003). In addition to cats it is also relevant to emphasise that dogs, foxes and raccoon dogs are primary hosts for zoonotic geohelminths (Toxocara spp. and Uncinaria stenocephala Railliet, 1884) present in Estonian rural and urban areas (Plumer et al. 2014, Laurimaa et al. 2016a,b, Tull et al. 2020).

We also recorded helminths that have only in rare cases infected humans, i.e. Hydatigera taeniaeformis, Ancylostoma tubaeforme and Eucoleus aerophilus (see Rossin et al. 2004, Altreuther et al. 2005, Lalosević et al. 2008). Our study found for the first time in Estonia A. tubaeforme in cat faecal samples; it is a widespread cat parasite throughout the world. Ancylostoma tubaeforme feeds on blood in the small intestine and heavy infections can lead to anemia and may be fatal for cats (Altreuther et al. 2005). Laurimaa et al. (2016b) found that $30 \%$ of raccoon dogs were infected with E. aerophilus. Moreover, majority of the examined red foxes $(87.6 \%)$ harboured that parasite (Laurimaa et al. 2016a). Our findings of T. cati, H. taeniaeformis, A. tubaeforme and E. aerophilus in cats from different locations, especially from rural areas, indicate coexisting sylvatic and synanthropic cycles, and imply a simple transmission route of zoonotic parasites between definitive and intermediate or reservoir hosts in the environment. Therefore, domestic cats, rather than wild carnivores, could pose a greater risk for humans because of their close contacts with humans. The lack of accurate diagnostics or infrequent diagnosis of the aforementioned endoparasites may result in higher numbers of infected humans. Therefore, health workers 
Table 2. Endoparasite (co)infection prevalence in cats $(\mathrm{N}=290)$ from Tartu Animal shelter according to their age, capture location, availability for adoption and sex. In each column, the first number indicates the number of infected cats $(\mathrm{N})$ and the second number represents the infection prevalence (\%). The minimum, maximum and mean intensities of endoparasite eggs per gram are shown in the last two columns.

\begin{tabular}{|c|c|c|c|c|c|c|c|c|c|c|c|}
\hline & \multicolumn{2}{|c|}{ Age } & \multicolumn{2}{|c|}{ Location } & \multicolumn{2}{|c|}{ Time } & \multicolumn{2}{|c|}{$\operatorname{Sex}^{1}$} & \multirow[b]{2}{*}{$\begin{array}{l}\text { Total fre- } \\
\text { quency, \% } \\
(\mathrm{N}=290)\end{array}$} & \multirow[b]{2}{*}{$\begin{array}{l}\text { Min-max } \\
\text { EPG/OPG }\end{array}$} & \multirow{2}{*}{$\begin{array}{c}\text { Mean } \\
\text { intensity } \\
\pm \text { standard } \\
\text { deviation } \\
\text { (EPG/ } \\
\text { OPG) }\end{array}$} \\
\hline & $\begin{array}{c}\text { Young } \\
(\mathrm{N}=120)\end{array}$ & $\begin{array}{c}\text { Adult } \\
(\mathrm{N}=170)\end{array}$ & $\begin{array}{c}\text { Rural } \\
(\mathrm{N}=160)\end{array}$ & $\begin{array}{c}\text { Urban** } \\
(\mathrm{N}=130)\end{array}$ & $\begin{array}{l}\text { Cats in } \\
\text { quarantine } \\
(\mathrm{N}=169)\end{array}$ & $\begin{array}{l}\text { Cats for } \\
\text { adoption } \\
(\mathrm{N}=121)\end{array}$ & $\begin{array}{c}\text { Female } \\
(\mathrm{N}=148)\end{array}$ & $\begin{array}{c}\text { Male } \\
(\mathrm{N}=104)\end{array}$ & & & \\
\hline Ancylostoma sp. & 0 & $2(1.2)$ & $2(1.3)$ & 0 & $2(1.2)$ & 0 & $2(1.4)$ & 0 & $\begin{array}{c}2 / 290 \\
(0.7)\end{array}$ & $80-340$ & $210 \pm 184$ \\
\hline Cryptosporidium spp. & $2(1.6)$ & $4(2.4)$ & $4(2.5)$ & $2(1.5)$ & $4(2.4)^{*}$ & $2(1.7)$ & $3(2.0)$ & $2(1.9)$ & $\begin{array}{l}6 / 290 \\
(2.1)\end{array}$ & $40-15,000$ & $\begin{array}{c}2,880 \pm \\
5,951\end{array}$ \\
\hline Eucoleus aerophilus & $2(1.6)$ & $4(2.4)$ & $5(3.1)$ & $1(0.8)$ & $6(3.6)^{*}$ & 0 & $4(2.7)$ & $0 *$ & $\begin{array}{l}6 / 290 \\
(2.1)\end{array}$ & 20 & 20 \\
\hline Giardia sp. & $1(0.8)$ & $1(0.6)$ & $2(1.3)$ & 0 & $2(1.2)$ & 0 & 0 & $2(1.9)$ & $\begin{array}{c}2 / 290 \\
(0.7)\end{array}$ & $20-120$ & $70 \pm 71$ \\
\hline Cystoisospora spp. & $14(11.7)$ & $22(12.9)$ & $23(14.4)$ & $13(10.0)$ & $12(7.1)^{* *}$ & $24(19.8)$ & $16(10.8)$ & $14(13.5)$ & $\begin{array}{l}36 / 290 \\
(12.4)\end{array}$ & $20-46,020$ & $\begin{array}{c}3,752 \pm \\
10,371\end{array}$ \\
\hline Taeniidae gen. sp. & $7(5.8)$ & $5(2.9)$ & $11(6.9)$ & $1(0.8)^{* *}$ & $10(5.9)$ & $2(1.7)$ & $4(2.7)$ & $4(3.9)$ & $\begin{array}{c}12 / 290 \\
(4.1)\end{array}$ & $20-300$ & $82 \pm 94$ \\
\hline Toxocara cati & $54(45.0)^{*}$ & $52(30.6)$ & $73(45.6)$ & $\begin{array}{c}33 \\
(25.4)^{* * *}\end{array}$ & $56(33.1)$ & $52(43.0)$ & $52(35.1)$ & $35(33.7)$ & $\begin{array}{c}106 / 290 \\
(36.6)\end{array}$ & $20-29,800$ & $\begin{array}{c}2,330 \pm \\
4,173\end{array}$ \\
\hline $\begin{array}{l}\text { Toxoplasma gondii/Hammon- } \\
\text { dia hammondi }\end{array}$ & $0 * *$ & $10(5.9)$ & $4(2.5)$ & $6(4.6)$ & $4(2.4)$ & $6(5.0)$ & $4(2.7)$ & $6(5.8)$ & $\begin{array}{c}10 / 290 \\
(3.4)\end{array}$ & $20-2,160$ & $374 \pm 694$ \\
\hline Total helminths & $63(52.5)^{*}$ & $63(37.2)$ & $91(56.9)$ & $\begin{array}{c}35 \\
(26.9)^{* * *}\end{array}$ & $74(43.8)$ & $52(43.0)$ & $62(41.9)$ & $39(37.5)$ & $\begin{array}{c}111 / 290 \\
(38.3)\end{array}$ & $20-29,800$ & $\begin{array}{c}2,019 \pm \\
3,950\end{array}$ \\
\hline Total protozoa & $17(14.2)$ & $37(21.8)$ & $33(20.6)$ & $21(16.2)$ & $22(13)^{*}$ & $32(26.5)$ & $23(15.5)$ & $22(21.2)$ & $\begin{array}{c}46 / 290 \\
(15.9)\end{array}$ & $20-46,020$ & $\begin{array}{c}2,893 \pm \\
8,744\end{array}$ \\
\hline T. cati + Cystoisospora spp. & $8(6.7)$ & $5(2.9)$ & $11(6.9)^{*}$ & $2(1.5)$ & $4(2.4)$ & $9(7.4)$ & $6(4.1)$ & $4(3.9)$ & $\begin{array}{c}13 / 290 \\
(4.5)\end{array}$ & $80-20,120$ & $\begin{array}{l}5,109 \pm \\
6,584\end{array}$ \\
\hline $\begin{array}{l}\text { Cystoisospora spp. }+ \text { T. gon- } \\
\text { dii/H. hammondi }\end{array}$ & 0 & $6(3.5)$ & $1(0.6)$ & $5(3.9)$ & $3(1.8)$ & $3(2.5)$ & $2(1.4)$ & $4(3.9)$ & $\begin{array}{l}6 / 290 \\
(2.1)\end{array}$ & $80-15,340$ & $\begin{array}{c}3,740 \pm \\
5,810\end{array}$ \\
\hline T. cati + E. aerophilus & $2(1.6)$ & $2(1.2)$ & $3(1.9)$ & $1(0.8)$ & $4(2.4)$ & 0 & $3(2.0)$ & 0 & $\begin{array}{l}4 / 290 \\
(1.4)\end{array}$ & $860-9,440$ & $\begin{array}{c}3,830 \pm \\
3,821\end{array}$ \\
\hline T. $c a t i+$ Taeniidae & $2(1.6)$ & $1(0.6)$ & $3(1.9)$ & 0 & $3(1.8)$ & 0 & $2(1.4)$ & 0 & $\begin{array}{c}3 / 290 \\
(1.0)\end{array}$ & $\begin{array}{c}2,340- \\
5,360\end{array}$ & $\begin{array}{l}3,453 \pm \\
1,659\end{array}$ \\
\hline $\begin{array}{l}\text { T. cati }+H . \text { taeniaeformis } \\
\text { ETC }\end{array}$ & $1(0.8)$ & $2(1.2)$ & $2(1.3)$ & $1(0.8)$ & $2(1.2)$ & $1(0.8)$ & $2(1.4)$ & $1(1.0)$ & $\begin{array}{l}3 / 290 \\
(1.0)\end{array}$ & $40-2,160$ & $\begin{array}{l}840 \pm \\
1,151\end{array}$ \\
\hline $\begin{array}{l}\text { T. cati }+ \text { Cryptosporidium } \\
\text { spp. + Taeniidae }+ \text { Cystoisos- } \\
\text { pora } \text { spp. }\end{array}$ & $1(0.8)$ & 0 & $1(0.6)$ & 0 & 0 & $1(0.8)$ & 0 & 0 & $\begin{array}{l}1 / 290 \\
(0.4)\end{array}$ & 46,020 & - \\
\hline $\begin{array}{l}\text { T. cati }+ \text { E. aerophilus }+H \text {. } \\
\text { taeniaeformis }\end{array}$ & 0 & $1(0.6)$ & $1(0.6)$ & 0 & $1(0.6)$ & 0 & 0 & 0 & $\begin{array}{l}1 / 290 \\
(0.4)\end{array}$ & 140 & - \\
\hline $\begin{array}{l}\text { T. cati }+ \text { Giardia } \text { sp. }+ \\
\text { Taeniidae }\end{array}$ & $1(0.8)$ & 0 & $1(0.6)$ & 0 & $1(0.6)$ & 0 & 0 & $1(1.0)$ & $\begin{array}{l}1 / 290 \\
(0.4)\end{array}$ & 8,280 & - \\
\hline $\begin{array}{l}\text { T. cati }+ \text { Cryptosporidium } \\
\text { spp. }\end{array}$ & $1(0.8)$ & 0 & $1(0.6)$ & 0 & $1(0.6)$ & 0 & $1(0.7)$ & 0 & $\begin{array}{l}1 / 290 \\
(0.4)\end{array}$ & 3,880 & - \\
\hline $\begin{array}{l}\text { Toxocara cati }+ \text { Toxoplasma } \\
\text { gondii/H. hammondi }\end{array}$ & 0 & $1(0.6)$ & $1(0.6)$ & 0 & 0 & $1(0.8)$ & $1(0.7)$ & 0 & $\begin{array}{l}1 / 290 \\
(0.4)\end{array}$ & 320 & - \\
\hline $\begin{array}{l}\text { Cryptosporidium spp. }+T \text {. } \\
\text { gondii/H. hammondi }\end{array}$ & 0 & $1(0.6)$ & $1(0.6)$ & 0 & 0 & $1(0.8)$ & 0 & $1(1.0)$ & $\begin{array}{l}1 / 290 \\
(0.4)\end{array}$ & 16,000 & - \\
\hline $\begin{array}{l}\text { Cystoisospora spp. + Taeni- } \\
\text { idae }\end{array}$ & $1(0.8)$ & 0 & $1(0.6)$ & 0 & $1(0.6)$ & 0 & 0 & $1(1.0)$ & $\begin{array}{l}1 / 290 \\
(0.4)\end{array}$ & 240 & - \\
\hline $\begin{array}{l}\text { T. cati }+ \text { Cystoisospora spp. } \\
+ \text { H. taeniaeformis }\end{array}$ & $1(0.8)$ & 0 & $1(0.6)$ & 0 & $1(0.6)$ & 0 & 0 & 0 & $\begin{array}{l}1 / 290 \\
(0.4)\end{array}$ & 2,680 & - \\
\hline $\begin{array}{l}\text { Giardia sp. }+ \text { T. gondii/H. } \\
\text { hammondi }\end{array}$ & 0 & $1(0.6)$ & $1(0.6)$ & 0 & $1(0.6)$ & 0 & 0 & $1(1.0)$ & $\begin{array}{l}1 / 290 \\
(0.4)\end{array}$ & 280 & - \\
\hline Total coinfection & $\begin{array}{c}18 / 120 \\
(15.0) \\
\end{array}$ & $\begin{array}{c}20 / 170 \\
(11.8) \\
\end{array}$ & $\begin{array}{c}29 / 160 \\
(18.1) \\
\end{array}$ & $\begin{array}{c}9 / 130 \\
(6.9)^{* *}\end{array}$ & $\begin{array}{c}22 / 169 \\
(13.0)\end{array}$ & $\begin{array}{c}16 / 121 \\
(13.2) \\
\end{array}$ & $\begin{array}{c}17 / 148 \\
(11.5) \\
\end{array}$ & $\begin{array}{c}13 / 104 \\
(12.5)\end{array}$ & $\begin{array}{c}38 / 290 \\
(13.6) \\
\end{array}$ & & \\
\hline
\end{tabular}

$\mathrm{p}<0.05^{*} ; \mathrm{p}<0.01 * * ; \mathrm{p}<0.001 * * * ;{ }^{1}$ mixed cages of both sexes were excluded

and officials need to highlight the possibility that stray cats distribute zoonotic endoparasites that are hazardous to human health.

Genetic analysis confirmed the presence of $H$. taeniaeformis, which can cause severe illness in humans (Ekanayake et al. 1999). Previously, only Valdmann et al. (2004) have demonstrated infections with $H$. taeniaeformis $(3 \%)$ in Estonian Eurasian lynx. This parasite is a cosmopolitan taeniid and it uses felines as definitive hosts. However, we could not identify all taeniid eggs genetically due to the degradation and thus we cannot rule out the presence species of Echinococcus Rudolphi, 1801 parasites in some cat samples. Recent studies in Estonia have demonstrated that $4 \%$ of wolves and $2.2 \%$ of urban dogs were infected with Echinococcus granulosus (Batsch, 1786) sensu lato (Moks et al. 2006, Laurimaa et al. 2015b), whereas $7.1 \%$ of urban and $31.5 \%$ of rural red foxes with Echinococcus multilocularis (Laurimaa et al. 2015a, 2016a). Since stray cats can prey on epidemiologically relevant intermediate hosts of $E$. multilocularis in Europe (Arvicola amphibius [Linnaeus], 
Table 3. Faecal samples from infected shelter cats $(\mathrm{N}=290)$ positive for one or more endoparasite (CL: $95 \%$ confidence limits) divided by predictor variables. In the column, the first number indicates the number of infected cats $(\mathrm{N})$, followed by prevalence $(\%)$ in parentheses, with the $95 \%$ confidence limits below.

\begin{tabular}{|c|c|c|c|c|c|c|c|c|c|}
\hline & \multicolumn{2}{|c|}{ Age } & \multicolumn{2}{|c|}{ Location } & \multicolumn{2}{|c|}{ Time } & \multicolumn{2}{|c|}{ Sex ${ }^{1}$} & \multirow{2}{*}{$\begin{array}{c}\text { Total } \\
(\mathrm{N}=290)\end{array}$} \\
\hline & $\begin{array}{c}\text { Young } \\
(\mathrm{N}=120)\end{array}$ & $\begin{array}{c}\text { Adult } \\
(\mathrm{N}=170)\end{array}$ & $\begin{array}{c}\text { Rural } \\
(\mathrm{N}=160)\end{array}$ & $\begin{array}{c}\text { Urban } \\
(\mathrm{N}=130)\end{array}$ & $\begin{array}{l}\text { Cats in quaran- } \\
\text { tine }(\mathrm{N}=169)\end{array}$ & $\begin{array}{l}\text { Cats for adop- } \\
\text { tion }(\mathrm{N}=121)\end{array}$ & $\begin{array}{c}\text { Female } \\
(\mathrm{N}=148)\end{array}$ & $\begin{array}{c}\text { Male } \\
(\mathrm{N}=104)\end{array}$ & \\
\hline \multirow{2}{*}{$\begin{array}{l}\text { Positive cats, } \\
N(\%), 95 \% \text { CL }\end{array}$} & $59(49.2)$ & $79(46.5)$ & $91(56.9)$ & $47(36.2)$ & $72(42.6)$ & $66(54.5)$ & $68(46.0)$ & $49(47.1)$ & $138(47.6)$ \\
\hline & $40.1-58.2$ & $38.9-54.0$ & $49.1-64.6$ & $27.8-44.5$ & $35.1-50.1$ & $45.5-63.5$ & $37.8-54.1$ & $37.4-56.9$ & $41.8-53.4$ \\
\hline \multirow{2}{*}{$\begin{array}{l}\text { One endoparasite } \\
\text { species }\end{array}$} & $41(34.2)$ & $59(34.7)$ & $62(38.8)$ & $38(29.2)$ & $50(29.6)$ & $50(41.3)$ & $51(34.5)$ & $36(34.6)$ & $100(34.5)$ \\
\hline & $25.6-42.8$ & $27.5-41.9$ & $31.1-46.4$ & $21.3-37.2$ & $22.6-36.5$ & $32.4-50.2$ & $26.7-42.2$ & $25.3-43.9$ & $29.0-40.0$ \\
\hline \multirow{2}{*}{$\begin{array}{l}\text { Two endoparasite } \\
\text { species }\end{array}$} & $15(12.5)$ & $19(11.2)$ & $25(15.6)$ & $9(6.9)$ & $19(11.2)$ & $15(12.4)$ & $17(11.2)$ & $12(11.5)$ & 34 (11.7) \\
\hline & $6.5-18.5$ & $6.4-16.0$ & $9.9-21.3$ & $2.5-11.3$ & $6.4-16.1$ & $6.4-18.4$ & $6.3-16.7$ & $5.3-17.8$ & $8.0-15.4$ \\
\hline \multirow{2}{*}{$\begin{array}{l}\text { Three endoparasite } \\
\text { species }\end{array}$} & $2(1.7)$ & $1(0.6)$ & $3(1.9)$ & 0 & $3(1.8)$ & 0 & 0 & $1(1.0)$ & $3(1.0)$ \\
\hline & $0-4.0$ & $0-1.8$ & $0-4.0$ & - & $0-3.8$ & - & - & $0-2.9$ & $0-2.2$ \\
\hline \multirow{2}{*}{ Four endoparasites } & $1(0.8)$ & 0 & $1(0.6)$ & 0 & 0 & $1(0.8)$ & 0 & 0 & $1(0.3)$ \\
\hline & $0-2.5$ & - & $0-1.9$ & - & - & $0-2.5$ & - & - & $0-1.0$ \\
\hline \multirow{2}{*}{$\begin{array}{l}\text { More than one } \\
\text { endoparasite species }\end{array}$} & $18(15.0)$ & $20(11.8)$ & $29(18.1)$ & $9(6.9)$ & $22(13.0)$ & $16(13.2)$ & $17(11.5)$ & $13(12.5)$ & $38(13.1)$ \\
\hline & $8.5-21.5$ & $6.9-16.7$ & $12.1-24.2$ & $2.5-11.3$ & $7.9-18.1$ & $7.1-19.3$ & $6.3-16.7$ & $6.0-19.0$ & $9.2-17.0$ \\
\hline
\end{tabular}

${ }^{1}$ mixed cages of both sexes were excluded

Microtus arvalis [Pallas], Myodes glareolus [Schreber]) (Vuitton et al. 2003), some of them are likely infected.

Although E. multilocularis infection in cats has never been studied in Estonia, cats are likely to have lower infection rate compared to foxes (Moks et al. 2005, Laurimaa et al. 2015a) and raccoon dogs (Laurimaa et al. 2015c). A study performed in rural settlements in the endemic areas of France revealed $0-30 \%$ prevalence of E. multilocularis in cats depending on the region and year (Knapp et al. 2018). In a recent study, Karamon et al. (2019) detected relatively high E. multilocularis prevalence $(14.3 \%)$ in shelter cats in Poland, whereas a large-scale study in Germany found low prevalence $(0.25 \%)$ (Dyachenko et al. 2008). However, their zoonotic significance of shelter cats is considered to be low, as cats have low worm establishment and low number of infective eggs (Kapel et al. 2006, Deplazes et al. 2011).

\section{Risk factors}

In the current study urban cats had lower infection with T. cati and with taeniids than rural cats. Also, rural cats had more coinfections with multiple endoparasites and were therefore more intensely infected than urban cats. Cats in rural areas have probably larger home ranges and more contacts with other stray cats (McGlade et al. 2003). Furthermore, they may more frequently prey on intermediate or paratenic hosts (mainly rodents and birds), which can facilitate transmission of endoparasites (Becker et al. 2012, Antolová et al. 2013, Strube et al. 2013, Lefkaditis et al. 2014). Nijsse et al. (2016) found that the more time a cat was allowed to roam outdoors individually, the higher was the risk of infection with $T$. cati. In Finland, Näreaho et al. (2012) found a much lower prevalence of T. cati (5.4\%) and Taenia sp. (1.5\%) in owned cats but the risk factors were similar to those of our study, including access to outdoors and living outside of cities. As most of the cats in this study were free-ranging, they had higher probability to be infected with endoparasites. A recent meta-analysis also confirmed that outdoor access is a significant risk factor for parasitic infection in cats across 19 different path- ogens including many relevant to human, domestic animal and wildlife health, such as T. gondii and T. cati (see Chalkowski et al. 2019).

A study in Hungary indicated that cats whose owners claim the use of anthelmintics were significantly less frequently helminth-positive than cats that were not dewormed (Capári et al. 2013). Another study by Beugnet et al. (2014) confirmed that cats receiving more than three treatments per year were significantly less infected than cats receiving one or two treatments per year. Mircean et al. (2010) showed that while a majority of urban cat owners applied anthelmintic treatment at least four times in a year, only a small proportion of rural cat owners gave one treatment in a year. For adult and young cats, individual risk assessments should be implemented by a form of decision tree which has been suggested by ESCCAP (2017). Current information indicates that once- or twice-yearly treatments do not significantly prevent infection (ESCCAP 2020). Therefore, treatment at least four times a year is a general recommendation if cats have access to the outdoors or are housed outside (ESCCAP 2017).

The infection with endoparasites remained considerably high among young and adult shelter cats after quarantine time. According to the infection intensity model, young cats had nearly three times higher infection intensity with endoparasites than adult cats. Young age has been identified by various authors as a serious risk factor for endoparasite infection in cats (Barutzki and Schaper 2003, Mircean et al. 2010, Zottler et al. 2019). Young shelter cats were intensely (co)infected with T. cati, Cystoisospora spp., Cryptosporidium spp. and taeniids but adults had in addition to T. cati and H. taeniaeformis decreased (co)infections with protozoan species (Cryptosporidium spp. and $T$. gondii/H. hammondi). Such a pattern may indicate a lack of immune response in young cats, especially in the case of $T$. cati, because it is acquired via lactogenic transmission (Coati et al. 2004). In contrast, stray cats are probably malnourished, hence, increasing their susceptibility to parasitic infections. Afterwards their nutritional intake increases in 
the animal shelter but cats remain endoparasite spreaders post-quarantine time, if not treated with anthelmintics.

We found that $T$. cati was most common in young cats, but adult cats had significant risk for the presence of $T$. gondii/H. hammondi. Unfortunately, we could not differentiate between oocysts of $T$. gondii and $H$. hammondi because of their similar size. As $H$. hammondi has not been recorded nor studied in Estonia, it is unclear whether these oocysts belonged to $T$. gondii or H. hammondi. However, various studies have shown that $T$. gondii is highly endemic in Estonian environment infecting cats as well as wildlife species like moose (Alces alces [Linnaeus]) and wild boar (Sus scrofa Linnaeus) (Jokelainen et al. 2015, Remes et al. 2018). Furthermore, several authors have indicated that the overall seroprevalence of $T$. gondii in cats is high (up to 60.8\%) and infection risk factors included older age in cats (Deksne et al. 2013, Must et al. 2015). Toxoplasma gondii is a zoonotic parasite that is a threat for immunocompromised people and for pregnant women (Dabritz and Conrad 2010). In general, cats may shed millions oocysts of $T$. gondii during one to three weeks (Dubey 1995), and cats of all ages can shed oocysts in nature (Dubey 2010). Furthermore, young cats can acquire endoparasite infection, including T. cati, from their mother via milk (vertical transmission) or through the contaminated environment (horizontal transmission, e.g., T. gondii/H. hammondi, Cryptosporidium sp., Giardia sp., Ancylostoma tubaeformae) (Fayer et al. 2000, Coati et al. 2004, Baneth et al. 2016).

\section{Protista}

As we could not identify all taxa to the species level, it is possible that cats were infected with zoonotic Giardia duodenalis assemblage A, Cryptosporidium felis or Cryptosporidium parvum Tyzzer, 1912 (Cacciò et al. 2002, Tzannes et al. 2008, Heyworth 2016). Therefore, further studies are needed in order to determine protozoan species. Although Cystoisospora spp. do not have zoonotic potential, they can cause gastrointestinal diseases in cats (Palmer et al. 2008).

Cats available for adoption had relatively high prevalence and intensities of protozoan parasites. Generally, animal shelters have limited resources and must accommodate large numbers of animals. A study by Schurer et al. (2015) identified cost as a major deciding factor in choosing anthelmintics. Due to cost-efficient managing, the shelters' policy foresees that protozoan infections are treated only when cats display clinical signs, and, therefore, some cats can remain infected with zoonotic protozoan taxa like $C$. felis and G. duodenalis assemblage A. However, the true prevalence of Cystoisospora spp., Cryptosporidium spp. and Giardia sp. may be even higher because thawing samples could reduce the detection rate. Moreover, the small dimensions of oocysts could impair the detection of the protozoan parasites using the McMaster technique
(McGlade et al. 2003) such that intermittent stages of protozoan parasites remain undetected.

Some shelter cats may experience stress-induced appetite loss and may not consume enough anthelmintic when mixed with food, facilitating endoparasite spread in the shelter. Thus, animal shelters with high endoparasite prevalence and intensity should re-evaluate their parasite control procedures (Spain et al. 2001, Villeneuve et al. 2015, Blasco et al. 2017, Zottler et al. 2019). Our study suggests that more attention should be paid to infection with protozoa and $T$. cati because infected cats may pose a threat not only to other shelter cats, but also to the shelter staff and new owners. Faecal samples should be taken frequently from quarantine cats and measures undertaken for adequate isolation, environmental hygiene and, in indicated cases, treatment to prevent parasite spread (ESCCAP 2018). Protocols and recommendations for the parasitic infection control should be communicated clearly to shelter staff and consistently applied (ESCCAP 2020).

\section{Recommendations for reducing the parasite burden in shelter}

Considering the relatively high parasite burden in shelter cats in Tartu, more effective anthelmintic treatment is required. Special attention should be paid to eliminate zoonotic parasites (e.g., T. cati), but also other endoparasites with high prevalence (e.g., Cystoisospora spp.). It is also important to control the numbers of cats in shelter to avoid overcrowding. Constant arrival of new cats can result in overcrowded conditions that promote parasite transmission. Palmer et al. (2008) argued that limited financial resources and poor cleaning may lead to insufficient anthelmintic treatment. The financial situation in animal shelters, including the Tartu Animal Shelter, should be improved to find better treatment and management against parasites to eliminate or at least reduce the infection intensity.

To conclude, our findings highlight that a high percentage of stray cats brought to the Tartu Animal Shelter are infected with endoparasites, including zoonotic agents, and remain infected after a quarantine period. Infected cats can transmit parasites to uninfected animals in the shelter and are a potential threat to shelter staff and people adopting cats. The results of this study emphasise the need to find and implement measures for more efficient control of parasites in animal shelters as well as in stray cats.

Acknowledgements. We are grateful to the staff of the Tartu Animal Shelter for their fruitful cooperation. We sincerely thank John Davison for helpful comments and suggestions. This work was supported by research funding from the Estonian Ministry of Education and Research (IUT20-32) and by a grant (PLTOM20905) from the Institute of Ecology and Earth Sciences, University of Tartu, Estonia. This work was supported by research funding (grant PRG1209) from the Estonian Ministry of Education and Research. 


\section{REFERENCES}

Altreuther G., Borgsteede F.H.M., Buch J., Charles S.D., Cruthers L., Epe C., Young D.R., Krieger K.J. 2005: Efficacy of a topically administered combination of emodepside and praziquantel against mature and immature Ancylostoma tubaeforme in domestic cats. Parasitol. Res. 97: S51-S57.

Antolová D., Reiterová K., Stanko M., Zalesny G., Fričová J., DvorožŇÁkovÁ E. 2013: Small mammals: paratenic hosts for species of Toxocara in eastern Slovakia. J. Helminthol. 87: $52-58$.

Baneth G., Thamsborg S.M., Otranto D., Guillot J., Blaga R., Deplazes P., Solano-Gallego L. 2016: Major parasitic zoonoses associated with dogs and cats in Europe. J. Comp. Pathol. 155: S54-S74.

BARTON K. 2019: Multi-Model Inference. R package version 1.43.6. https://CRAN.R-project.org/package=MuMIn

BARUTZKi D., SCHAPER R. 2003: Endoparasites in dogs and cats in Germany 1999-2002. Parasitol Res. 90: S148-S150.

Becker A.C., Rohen M., Epe C., Schnieder T. 2012: Prevalence of endoparasites in stray and fostered dogs and cats in northern Germany. Parasitol Res. 111: 849-857.

Beugnet F., Bourdeau P., Chalvet-Monfray K., Cozma V., Farkas R., Guillot J., Halos L., Joachim A., Losson B., Miró G., Otranto D., Renaud M., Rinaldi L. 2014: Parasites of domestic owned cats in Europe: co-infestations and risk factors. Parasit. Vectors 7: 291.

Blasco X., Salas A., Manuelian C.L., Torre C., Ortuño A. 2017: Intestinal parasitic infection in multi-cat shelters in catalonia. Refu. Vet. 72: 16-21.

Brooks E.M., Kristensen K., Benthem J. K., Magnusson A., Berg W.C., Nielsen A., Skaug J.H., Maechler M., BolkER M.B. 2017: glmmTMB balances speed and flexibility among packages for zero-inflated generalized linear mixed modeling. $\mathrm{R}$ Journal 9: 378-400.

Burnham K.P., Anderson D.R. (Eds.) 2004: Model Selection and Multimodel Inference. Springer, New York, $515 \mathrm{pp}$.

Cacciò S., Pinter E., Fantini R., Mezzaroma I., Pozio E. 2002: Human Infection with Cryptosporidium felis: case report and literature review. Emerg. Infect. Dis. 8: 85-86.

Capári B., Hamel D., Visser M., Winter R., Pfister K., RehbeIn S. 2013: Parasitic infections of domestic cats, Felis catus, in western Hungary. Vet. Parasitol. 192: 33-42.

Chalkowski K., Wilson A.E., Lepczyk C.A., Zohdy S. 2019: Who let the cats out? A global meta-analysis on risk of parasitic infection in indoor versus outdoor domestic cats (Felis catus). Biol. Lett. 15: 20180840

Coati N., Schnieder T., Epe C. 2004: Vertical transmission of Toxocara cati Schrank 1788 (Anisakidae) in the cat. Parasitol. Res. 92: 142-146.

Dabritz H.A., Conrad P.A. 2010: Cats and Toxoplasma: implications for public health. Zoonoses Public Health 57: 34-52.

Deksne G., Petrusēviča A., Kirjušina M. 2013: Seroprevalence and factors associated with Toxoplasma gondii infection in domestic cats from urban areas in Latvia. J. Parasitol. 99: 48-50.

Deplazes P., Hegglin D., Gloor S., Romig T. 2004: Wilderness in the city: the urbanization of Echinococcus multilocularis. Trends Parasitol. 20: 77-84.

Deplazes P., van Knapen F., Schweiger A., Overgaauw P.A.M. 2011: Role of pet dogs and cats in the transmission of helminthic zoonoses in Europe, with a focus on echinococcosis and toxocarosis. Vet. Parasitol. 182: 41-53.

Despommier D. 2003: Toxocariasis: clinical aspects, epidemiology, medical ecology, and molecular aspects. Clin. Microbiol. Rev. 16: 265-272.

Dubey J.P. 1995: Duration of immunity to shedding of Toxoplasma gondii oocysts by cats. J. Parasitol. 81: 410.
Dubey J. 2010: Toxoplasmosis of Animals and Humans. Second Edition. CRC Press, Boca Raton, 313 pp.

Dubey J.P. 2018: A review of Cystoisospora felis and C. rivolta-induced coccidiosis in cats. Vet. Parasitol. 263: 34-48.

Dyachenko V., Pantchev N., Gawlowska S., Vrhovec M.G., BAUER C. 2008: Echinococcus multilocularis infections in domestic dogs and cats from Germany and other European countries. Vet. Parasitol. 157: 244-253.

Ekanayake S., Warnasuriya N.D., Samarakoon P.S., Abewickrama H., Kuruppuarachchi N.D., Dissanaike A.S. 1999: An unusual 'infection' of a child in Sri Lanka, with Taenia taeniaeformis of the cat. Ann. Trop. Med. Parasitol. 93: 869-873.

ESCCAP: Control of Intestinal Protozoa in Dogs and CATS. 2018. European Scientific Counsel Companion Animal Parasites. Second Edition. Malvern Hills Science Park, Malvern, $22 \mathrm{pp}$.

ESCCAP: Worm Control in Dogs and Cats. 2017. European Scientific Counsel Companion Animal Parasites. Third Edition. Malvern Hills Science Park, Malvern, 41 pp.

ESCCAP: Worm Control in Dogs and CATS. 2020. European Scientific Counsel Companion Animal Parasites. Sixth Edition. Malvern Hills Science Park, Malvern, 42 pp.

Fayer R., Morgan U., Upton S.J. 2000: Epidemiology of Cryptosporidium: transmission, detection and identification. Int. J. Parasitol. 30: 1305-1322.

Gates M.C., Nolan T.J. 2009: Endoparasite prevalence and recurrence across different age groups of dogs and cats. Vet. Parasitol. 166: 153-158.

Gracenea M., Gómez M., Torres J. 2009: Prevalence of intestinal parasites in shelter dogs and cats in the metropolitan area of Barcelona (Spain). Acta Parasitol. 54: 73-77.

Greenwood J.S. 2020. Protozoan Parasites of Veterinary importance. 2020: http://people.upei.ca/sgreenwood/html/protozoa. html

Heinze G., Ploner M. 2018: logistf: Firth's Bias-Reduced Logistic Regression. R package version 1.23. https://CRAN.R-project. org/package $=$ logistf

Heyworth M.F. 2016: Giardia duodenalis genetic assemblages and hosts. Parasite 23: 13.

Jokelainen P., Simola O., Rantanen E., Näreaho A., Lohi H., Sukura A. 2012: Feline toxoplasmosis in Finland: cross-sectional epidemiological study and case series study. J. Vet. Diagn. Invest. 24: 1115-1124.

Jokelainen P., Velström K., Lassen B. 2015: Seroprevalence of Toxoplasma gondii in free-ranging wild boars hunted for human consumption in Estonia. Acta Vet. Scand. 57: 42.

Kapel C.M.O., Torgerson P.R., Thompson R.C.A., Deplazes P. 2006: Reproductive potential of Echinococcus multilocularis in experimentally infected foxes, dogs, raccoon dogs and cats. Int. J. Parasitol. 36: 79-86.

Karamon J., Sroka J., Dąbrowska J., Bilska-Zając E., Zdybel J., Kochanowski M., Różycki M., Cencek T. 2019: First report of Echinococcus multilocularis in cats in Poland: a monitoring study in cats and dogs from a rural area and animal shelter in a highly endemic region. Parasit. Vectors 12: 313.

Khatat S., Rosenberg D., Benchekroun G., Polack B. 2016: Lungworm Eucoleus aerophilus (Capillaria aerophila) infection in a feline immunodeficiency virus-positive cat in France. JFMS Open Rep. 2: 1-5.

Knapp J., Giraudoux P., Combes B., Umhang G., Boué F., Said-Ali Z., Aknouche S., Garcia C., Vacheyrou M., Laboissière A., Raton V., Comte S., Favier S., Demerson J.-M., Caillot C., Millon L., Raoul F. 2018: Rural and urban distribution of wild and domestic carnivore stools in the con- 
text of Echinococcus multilocularis environmental exposure. Int. J. Parasitol. 48: 937-946.

Kostopoulou D., Claerebout E., Arvanitis D., Ligda P., Voutzourakis N., Casaert S., Sotiraki S. 2017: Abundance, zoonotic potential and risk factors of intestinal parasitism amongst dog and cat populations: the scenario of Crete, Greece. Parasit. Vectors 10: 43.

Lalošević D., Stanojev-Jovanović D., Lalošević V., Pozio E., KLEM I. 2008: Pulmonary capillariasis miming bronchial carcinoma. Am. J. Trop. Med. Hyg. 78: 14-16.

Lassen B., Janson M., Viltrop A., Neare K., Hütt P., Golovljova I., Tummeleht L., Jokelainen P. 2016: Serological evidence of exposure to globally relevant zoonotic parasites in the estonian population. PLoS One 11: e 0164142.

Laurimaa, L., Davison, J., Plumer, L., Süld, K., Oja, R., Moks, E., Keis, M., Hindrikson, M., Kinkar L., Laurimäe T., Abner J., Remm J., Anijalg P., Saarma U. 2015a: Noninvasive detection of Echinococcus multilocularis tapeworm in urban area, Estonia. Emerg. Infect. Dis. 21: 163-164.

Laurimaa L., Davison J., Süld K., Plumer L., Oja R., Moks E., Keis M., Hindrikson M., Kinkar L., Laurimäe T., AвNer J., Remm J., Anijalg P., SaArma U. 2015b: First report of highly pathogenic Echinococcus granulosus genotype G1 in dogs in a European urban environment. Parasit. Vectors 8: 182.

Laurimaa L., Moks E., Soe E., Valdmann H., Saarma U. 2016a: Echinococcus multilocularis and other zoonotic parasites in red foxes in Estonia. Parasitology 143: 1450-1458.

Laurimaa L., Süld K., Davison J., Moks E., Valdmann H., SaARma U. 2016b: Alien species and their zoonotic parasites in native and introduced ranges: The raccoon dog example. Vet. Parasitol. 219: 24-33.

Laurimaa L., Süld K., Moks E., Valdmann H., Umhang G., Knapp J., SaArma U. 2015c: First report of the zoonotic tapeworm Echinococcus multilocularis in raccoon dogs in Estonia, and comparisons with other countries in Europe. Vet. Parasitol. 212: 200-205.

Lefkaditis M., Paştiu A., Rodi-Buriel A., Sossidou A., Panorias A., Eleftheriadis T., Cozma V., Mihalca A. 2014: Helminth burden in stray cats from Thessaloniki, Greece. Helminthologia 51: 73-76.

Leoni F., Gallimore C.I., Green J., McLauchlin J. 2003: Molecular epidemiological analysis of Cryptosporidium isolates from humans and animals by using a heteroduplex mobility assay and nucleic acid sequencing based on a small double-stranded RNA element. J. Clin. Microbiol. 41: 981-992.

Loftin C.M., Donnett U.B., Schneider L.G., Varela-Stokes A.S. 2019: Prevalence of endoparasites in northern Mississippi shelter cats. Vet. Parasitol. Reg. Stud. Reports 18: 100322.

McGlade T.R., Robertson I.D., Elliot A.D., Read C., THOMPSON R.C.A. 2003: Gastrointestinal parasites of domestic cats in Perth, Western Australia. Vet. Parasitol. 117: 251-262.

Mircean V., Titilincu A., Vasile C. 2010: Prevalence of endoparasites in household cat (Felis catus) populations from Transylvania (Romania) and association with risk factors. Vet. Parasitol. 171: 163-166.

Moks E., Jõgisalu I., Saarma U., Talvik H., Järvis T., ValdMANN H. 2006: Helminthological survey of wolf (Canis lupus) in Estonia, with an emphasis on the occurrence of Echinococcus granulosus. J. Wildl. Dis. 42: 359-365.

Moks E., Saarma U., Valdmann H. 2005: Echinococcus multilocularis in Estonia. Emerg. Infect. Dis. 11: 1973-1974.

Must K., Lassen B., Jokelainen P. 2015: Seroprevalence of and risk factors for Toxoplasma gondii infection in cats in Estonia. Vector Borne Zoonotic Dis. 15: 597-601.

Näreaho A., Puomio J., SaArinen K., Jokelainen P., Juselius T., Sukura A. 2012: Feline intestinal parasites in Finland: prevalence, risk factors and anthelmintic treatment practices. J. Feline Med. Surg. 14: 378-383.

Nijsse R., Ploeger H.W., Wagenaar J.A., Mughini-Gras L. 2016: Prevalence and risk factors for patent Toxocara infections in cats and cat owners' attitude towards deworming. Parasitol Res. 115: 4519-4525.

Otranto D., Cantacessi C., Pfeffer M., Dantas-Torres F., Brianti E., Deplazes P., Genchi C., Guberti V., Capelli G. 2015: The role of wild canids and felids in spreading parasites to dogs and cats in Europe. Vet. Parasitol. 213: 12-23.

Overgaaum P.A.M., van Zutphen L., Hoek D., Yaya F.O., Roelfsema J., Pinelli E., van Knapen F., Kortbeek L.M. 2009: Zoonotic parasites in fecal samples and fur from dogs and cats in The Netherlands. Vet. Parasitol. 163: 115-122.

Palmer C.S., Thompson R.C.A., Traub R.J., Rees R., RobertSON I.D. 2008: National study of the gastrointestinal parasites of dogs and cats in Australia. Vet. Parasitol. 151: 181-190.

Pavlásek I., Ryan U. 2007: The first finding of a natural infection of Cryptosporidium muris in a cat. Vet. Parasitol. 144: 349-352.

Plumer L., Davison J., SaArma U. 2014: Rapid urbanization of red foxes in Estonia: distribution, behaviour, attacks on domestic animals, and health-risks related to zoonotic diseases. PLoS One 9: e115124.

R Core Team 2020: R: A language and environment for statistical computing. $\mathrm{R}$ foundation for statistical computing, Vienna. https://www.r-project.org/

Remes N., Kärssin A., Must K., Tagel M., Lassen B., JoKelaINEN P. 2018: Toxoplasma gondii seroprevalence in free-ranging moose (Alces alces) hunted for human consumption in Estonia: indicator host species for environmental Toxoplasma gondii oocyst contamination. Vet. Parasitol. Reg. Stud. Reports 11: $6-11$.

Roepstorff A., Nansen P. 1998: The Epidemiology, Diagnosis and Control of Helminth Parasites of Swine, FAO Animal Health Manual. Food and Agriculture Organization of the United Nations, Rome, $171 \mathrm{pp}$.

Rossin A., Malizia A.I., Denegri G.M. 2004: The role of the subterranean rodent Ctenomys talarum (Rodentia: Octodontidae) in the life cycle of Taenia taeniaeformis (Cestoda: Taeniidae) in urban environments. Vet. Parasitol. 122: 27-33.

Saarma U., Jõgisalu I., Moks E., Varcasia A., Lavikainen A., Oksanen A., Simsek S., Andresiuk V., Denegri G., González L.M., Ferrer E., Gárate T., Rinaldi L., MarAVILLA P. 2009: A novel phylogeny for the genus Echinococcus, based on nuclear data, challenges relationships based on mitochondrial evidence. Parasitology 136: 317-328.

Schurer J.M., McKenzie C., Dowling P.M., Bouchard E., Jenkins E.J. 2015: Parasite control in Canadian companion animal shelters and a cost-comparison of anthelmintics. Can. Vet. J. 56: 964-970.

Schurer J.M., Ndao M., Skinner S., Irvine J., Elmore S.A., Epp T., Jenkins E.J. 2013: Parasitic zoonoses: one health surveillance in northern Saskatchewan. PLoS Negl. Trop. Dis. 7: e2141.

Spain C.V., Scarlett J.M., Wade S.E., McDonough P. 2001: Prevalence of enteric zoonotic agents in cats less than 1 year old in central New York State. J. Vet. Intern. Med. 15: 33-38.

Strube C., Heuer L., Janecek E. 2013: Toxocara spp. infections in paratenic hosts. Vet. Parasitol. 193: 375-389.

Suzuki J., Murata R., Kobayashi S., Sadamasu K., Kai A., TAKEUCHI T. 2011: Risk of human infection with Giardia $d u$ odenalis from cats in Japan and genotyping of the isolates to assess the route of infection in cats. Parasitology 138: 493-500.

Talvik H., Moks E., Mägi E., Järvis T., Miller I. 2006: Distribution of Toxocara infection in the environment and in definitive and paratenic hosts in Estonia. Acta Vet. Hung. 54: 399-406.

Tartu Animal Shelter 2019: Statistics of Tartu Animal Shelter 2019. https://www.loomadevarjupaik.eu/en/varjupaik/statistika.

Tokiwa T., Ohnuki A., Kubota R., Tamukai K., Ike K. 2018: Morphological and molecular characterization of Cystoisospora sp. from Asian small-clawed otters Aonyx cinereus. Int. J. Parasitol. Parasit. Wildl. 7: 268-273. 
Tull A., Moks E., Laurimaa L., Keis M., Süld K. 2020: Endoparasite infection hotspots in Estonian urban areas. J. Helminthol. 94: 1-9.

Tzannes S., Batchelor D.J., Graham P.A., Pinchbeck G.L., Wastling J., German A.J. 2008: Prevalence of Cryptosporidium, Giardia and Isospora species infections in pet cats with clinical signs of gastrointestinal disease. J. Feline Med. Surg. 10: $1-8$.

Valdmann H., Moks E., Talvik H. 2004: Helminth fauna of Eurasian lynx (Lynx lynx) in Estonia. J. Wildl. Dis. 40: 356-360.

Villeneuve A., Polley L., Jenkins E., Schurer J., Gilleard J., Kutz S., Conboy G., Benoit D., Seewald W., Gagné F.
2015: Parasite prevalence in fecal samples from shelter dogs and cats across the Canadian provinces. Parasit. Vectors 8: 281.

Vuitton D.A., Zhou H., Bresson-Hadni S., Wang Q., Piarroux M., Raoul F., Giraudoux P. 2003: Epidemiology of alveolar echinococcosis with particular reference to China and Europe. Parasitology 127: S87-S107.

Wyrosdick H.M., Chapman A., Martinez J., Schaefer, J.J. 2017: Parasite prevalence survey in shelter cats in Citrus County, Florida. Vet. Parasitol. Reg. Stud. Reports. 10: 20-24.

Zottler E.-M., Bieri M., Basso W., Schnyder M. 2019: Intestinal parasites and lungworms in stray, shelter and privately owned cats of Switzerland. Parasitol. Int. 69: 75-81.

Cite this article as: Tull A., Moks E., Saarma U. 2021: Endoparasite prevalence and infection risk factors among cats in an animal shelter in Estonia. Folia Parasitol. 68: 010. 